\title{
FEW RELATIONS ON THE GROWTH RATES OF COMPOSITE ENTIRE FUNCTIONS USING THEIR $(p, q)$ TH ORDER
}

\author{
SANJIB KUMAR DATTA, TANMAY BISWAS AND MANAB BISWAS
}

\begin{abstract}
In this paper we discuss the growth rates of the maximum term of composition of entire functions with their corresponding left and right factors.
\end{abstract}

\section{Introduction, definitions and notations}

Let $f$ be an entire function defined in the open complex plane $\mathbb{C}$. The maximum term $\mu(r, f)$ of $f=\sum_{n=0}^{\infty} a_{n} z^{n}$ on $|z|=r$ is defined by $\mu(r, f)=\max _{n \geq 0}\left(\left|a_{n}\right| r^{n}\right)$. We do not explain the standard definitions and notations in the theory of entire function as those are available in [6] In the sequel the following two notations are used:

$$
\begin{aligned}
& \log ^{[k]} x=\log \left(\log ^{[k-1]} x\right) \text { for } k=1,2,3, \cdots ; \\
& \log ^{[0]} x=x
\end{aligned}
$$

and

$$
\begin{aligned}
& \exp ^{[k]} x=\exp \left(\exp ^{[k-1]} x\right) \text { for } k=1,2,3, \cdots ; \\
& \exp ^{[0]} x=x
\end{aligned}
$$

To start our paper we just recall the following definitions:

Definition 1. The order $\rho_{f}$ and lower order $\lambda_{f}$ of an entire function $f$ is defined as follows:

$$
\rho_{f}=\limsup _{r \rightarrow \infty} \frac{\log ^{[2]} M(r, f)}{\log r} \text { and } \lambda_{f}=\liminf _{r \rightarrow \infty} \frac{\log ^{[2]} M(r, f)}{\log r} \text {. }
$$

Definition 2 ([3]). Let $l$ be an integer $\geq 2$. The generalised order $\rho_{f}^{[l]}$ and generalised lower order $\lambda_{f}^{[l]}$ of an entire function $f$ are defined as

$$
\rho_{f}^{[l]}=\limsup _{r \rightarrow \infty} \frac{\log ^{[l]} M(r, f)}{\log r} \text { and } \lambda_{f}^{[l]}=\liminf _{r \rightarrow \infty} \frac{\log ^{[l]} M(r, f)}{\log r} .
$$

When $l=2$, Definition 2 coincides with Definition 1 .

Corresponding author: Sanjib Kumar Datta.

2010 Mathematics Subject Classification. 30D30, 30D35.

Key words and phrases. order, lower order, $(p, q)$ th order, $(p, q)$ th lower order, entire function, maximum term, composition, growth. 
Juneja, Kapoor and Bajpai [2] defined the $(p, q)$ th order and $(p, q)$ th lower order of an entire function $f$ respectively as follows:

$$
\rho_{f}(p, q)=\limsup _{r \rightarrow \infty} \frac{\log ^{[p]} M(r, f)}{\log ^{[q]} r} \text { and } \lambda_{f}(p, q)=\liminf _{r \rightarrow \infty} \frac{\log ^{[p]} M(r, f)}{\log ^{[q]} r},
$$

where $p, q$ are positive integers with $p>q$.

For $p=2$ and $q=1$ we respectively denote $\rho_{f}(2,1)$ and $\lambda_{f}(2,1)$ by $\rho_{f}$ and $\lambda_{f}$.

Since for $0 \leq r<R$,

$$
\mu(r, f) \leq M(r, f) \leq \frac{R}{R-r} \mu(R, f)\{c f .[5]\}
$$

it is easy to see that

$$
\begin{array}{cc}
\rho_{f}=\limsup _{r \rightarrow \infty} \frac{\log ^{[2]} \mu(r, f)}{\log r}, & \lambda_{f}=\liminf _{r \rightarrow \infty} \frac{\log ^{[2]} \mu(r, f)}{\log r} ; \\
\rho_{f}^{[l]}=\limsup _{r \rightarrow \infty} \frac{\log ^{[l]} \mu(r, f)}{\log r}, & \lambda_{f}^{[l]}=\liminf _{r \rightarrow \infty} \frac{\log ^{[l]} \mu(r, f)}{\log r} ;
\end{array}
$$

and

$$
\rho_{f}(p, q)=\limsup _{r \rightarrow \infty} \frac{\log ^{[p]} \mu(r, f)}{\log ^{[q]} r}, \quad \lambda_{f}(p, q)=\liminf _{r \rightarrow \infty} \frac{\log ^{[p]} \mu(r, f)}{\log ^{[q]} r} .
$$

In this paper we wish to prove some results relating to the growth rates of maximum terms of composition of two entire functions with their corresponding left and right factors on the basis of $(p, q)$ th order and $(p, q)$ th lower order where $p, q$ are positive integers with $p>q$.

\section{Lemmas}

In this section we present some lemmas which will be needed in the sequel.

Lemma 1 ([4]). Let $f$ and $g$ be any two entire functions with $g(0)=0$. Then for all sufficiently large values of $r$,

$$
\mu(r, f \circ g) \geq \frac{1}{2} \mu\left(\frac{1}{8} \mu\left(\frac{r}{4}, g\right)-|g(0)|, f\right) .
$$

Lemma 2 ([1]). If $f$ and $g$ are any two entire functions then for all sufficiently large values of $r$,

$$
M(r, f \circ g) \leq M(M(r, g), f) .
$$

Lemma 3. Let $f$ be an entire function with non zero finite generalised order $\rho_{f}^{[l]}$ (non zero finite generalised lower order $\left.\lambda_{f}^{[l]}\right)$. If $p-q=l-1$, then the $(p, q)$ - th order $\rho_{f}(p, q)(\operatorname{lower}(p, q)-t h$ $\left.\operatorname{order} \lambda_{f}(p, q)\right)$ of $f$ will be equal to 1 . If $p-q \neq l-1$ then $\rho_{f}(p, q)\left(\lambda_{f}(p, q)\right)$ is either zero or infinity. 
Proof. From the definition of generalised order of an entire function $f$ we have for all sufficiently large values of $r$,

$$
\log ^{[l]} \mu(r, f) \leq\left(\rho_{f}^{[l]}+\varepsilon\right) \log r
$$

and for a sequence of values of $r$ tending to infinity,

$$
\log ^{[l]} \mu(r, f) \geq\left(\rho_{f}^{[l]}-\varepsilon\right) \log r
$$

Next let $a$ and $b$ be any two positive integers.

Now from (2) we have for all sufficiently large values of $r$,

$$
\log ^{[l+a]} \mu(r, f) \leq \log ^{[1+a]} r+O(1) \quad \text { i.e., } \quad \frac{\log ^{[l+a]} \mu(r, f)}{\log ^{[1+b]} r} \leq \frac{\log ^{[1+a]} r+O(1)}{\log ^{[1+b]} r}
$$

If we take $l+a=p$ and $1+b=q$, then $p-q=(l-1)+(a-b)$.

We discuss the following three cases:

Case I. Let $a=b$. Then from (4) we get for all sufficiently large values of $r$,

$$
\frac{\log ^{[p]} \mu(r, f)}{\log ^{[q]} r} \leq 1+\frac{O(1)}{\log ^{[1+a]} r} \quad \text { i.e., } \quad \limsup _{r \rightarrow \infty} \frac{\log ^{[p]} \mu(r, f)}{\log ^{[q]} r} \leq 1 .
$$

Similarly from (3) we have for a sequence of values of $r$ tending to infinity,

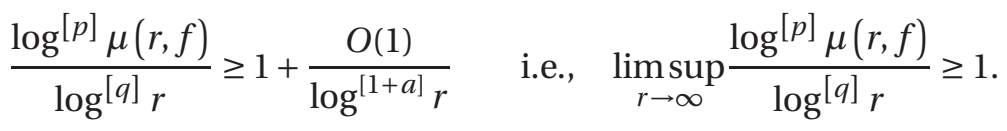

Now from(5) and (6) we have

$$
\rho_{f}(p, q)=1 \text { when } p-q=l-1 \text {. }
$$

Case II. Let $a>b$ (i.e., $\quad p-q \neq l-1$ ). Then from (4) we have for all sufficiently large values of $r$,

$$
\limsup _{r \rightarrow \infty} \frac{\log ^{[p]} \mu(r, f)}{\log ^{[q]} r} \leq 0 \quad \text { i.e., } \quad \rho_{f}(p, q)=0 \text { when } p-q \neq l-1 .
$$

Case III. Also let us choose $a$ and $b$ such that $a<b$ and $l+a>1+b$ (i.e., $\quad p-q \neq l-1$ ). Then from (3) it can be proved for a sequence of values of $r$ tending to infinity that

$$
\limsup _{r \rightarrow \infty} \frac{\log ^{[p]} \mu(r, f)}{\log ^{[q]} r} \geq \infty \quad \text { i.e., } \quad \rho_{f}(p, q)=\infty \text { when } p-q \neq l-1 .
$$

Therefore combining Case II and Case III (not violating the condition $p>q$ ), it follows that $\rho_{f}(p, q)$ is either zero or infinity. 
Similarly we may prove the conclusion for $\lambda_{f}(p, q)$.

This proves the lemma.

\section{Main results}

In this section we present the main results of the paper.

Theorem 1. Let $f$ and $g$ be any two entire functions such that $\rho_{g}(m, n)<\lambda_{f}(p, q) \leq \rho_{f}(p, q)<$ $\infty$, where $p, q, m, n$ are positive integers with $p>q, m>n$. Then

$$
\lim _{r \rightarrow \infty} \frac{\log [p] \mu\left(\exp ^{[n-1]} r, f \circ g\right)}{\log ^{[p-1]} \mu\left(\exp ^{[q-1]} r, f\right)}=0 \quad \text { if } q \geq m
$$

and

(ii) $\quad \lim _{r \rightarrow \infty} \frac{\log ^{[p+m-q-1]} \mu\left(\exp ^{[n-1]} r, f \circ g\right)}{\log ^{[p-1]} \mu\left(\exp ^{[q-1]} r, f\right)}=0 \quad$ if $q<m$.

Proof. Since $\rho_{g}(m, n)<\lambda_{f}(p, q)$, we can choose $\varepsilon(>0)$ is such a way that

$$
\rho_{g}(m, n)+\varepsilon<\lambda_{f}(p, q)-\varepsilon
$$

Now in view of the inequality (1),we have from Lemma 2 for all sufficiently large values of $r$,

$$
\begin{aligned}
\mu(r, f \circ g) & \leq M(r, f \circ g) \leq M(M(r, g), f) \\
\text { i.e., } \quad \log [p] \mu\left(\exp ^{[n-1]} r, f \circ g\right) & \leqslant \log [p] M\left(M\left(\exp ^{[n-1]} r, g\right), f\right) \\
\text { i.e., } \quad \log [p] \mu\left(\exp ^{[n-1]} r, f \circ g\right) & \leqslant\left(\rho_{f}(p, q)+\varepsilon\right) \log ^{[q]} M\left(\exp ^{[n-1]} r, g\right) .
\end{aligned}
$$

Now the following two cases may arise:

\section{Case I. Let $q \geqslant m$.}

Then we have from (8) for all sufficiently large values of $r$,

$$
\log ^{[p]} \mu\left(\exp ^{[n-1]} r, f \circ g\right) \leqslant\left(\rho_{f}(p, q)+\varepsilon\right) \log ^{[m-1]} M\left(\exp ^{[n-1]} r, g\right) .
$$

Again for all sufficiently large values of $r$,

$$
\begin{aligned}
\log ^{[m]} M\left(\exp ^{[n-1]} r, g\right) & \leqslant\left(\rho_{g}(m, n)+\varepsilon\right) \log { }^{[n]} \exp ^{[n-1]} r \\
\text { i.e., } \quad \log ^{[m]} M\left(\exp ^{[n-1]} r, g\right) & \leqslant\left(\rho_{g}(m, n)+\varepsilon\right) \log r \\
\text { i.e., } \quad \log ^{[m]} M\left(\exp ^{[n-1]} r, g\right) & \leqslant \log r^{\left(\rho_{g}(m, n)+\varepsilon\right)} \\
\text { i.e., } \quad \log ^{[m-1]} M\left(\exp ^{[n-1]} r, g\right) & \leqslant r^{\left(\rho_{g}(m, n)+\varepsilon\right)} .
\end{aligned}
$$

Now from (9) and (10) we have for all sufficiently large values of $r$,

$$
\log ^{[p]} \mu\left(\exp ^{[n-1]} r, f \circ g\right) \leqslant\left(\rho_{f}(p, q)+\varepsilon\right) r^{\left(\rho_{g}(m, n)+\varepsilon\right)} .
$$


Case II. Let $q<m$.

Then for all sufficiently large values of $r$ we get from (8) that

$$
\log { }^{[p]} \mu\left(\exp ^{[n-1]} r, f \circ g\right) \leqslant\left(\rho_{f}(p, q)+\varepsilon\right) \exp ^{[m-q]} \log ^{[m]} M\left(\exp ^{[n-1]} r, g\right) .
$$

Again for all sufficiently large values of $r$,

$$
\begin{aligned}
\log ^{[m]} M\left(\exp ^{[n-1]} r, g\right) & \leqslant\left(\rho_{g}(m, n)+\varepsilon\right) \log { }^{[n]} \exp ^{[n-1]} r \\
\text { i.e., } \quad \log ^{[m]} M\left(\exp ^{[n-1]} r, g\right) & \leqslant\left(\rho_{g}(m, n)+\varepsilon\right) \log r \\
\text { i.e., } \quad \log ^{[m]} M\left(\exp ^{[n-1]} r, g\right) & \leqslant \log r^{\rho_{g}(m, n)+\varepsilon} \\
\text { i.e., } \quad \exp ^{[m-q]} \log ^{[m]} M\left(\exp ^{[n-1]} r, g\right) & \leqslant \exp ^{[m-q]} \log r^{\rho_{g}(m, n)+\varepsilon} \\
\text { i.e., } \quad \exp ^{[m-q]} \log ^{[m]} M\left(\exp ^{[n-1]} r, g\right) & \leqslant \exp ^{[m-q-1]} r^{\rho_{g}(m, n)+\varepsilon} .
\end{aligned}
$$

Now from (12) and (13) we have for all sufficiently large values of $r$,

$$
\begin{aligned}
& \log { }^{[p]} \mu\left(\exp ^{[n-1]} r, f \circ g\right) \leq\left(\rho_{f}(p, q)+\varepsilon\right) \exp ^{[m-q-1]} r^{\rho_{g}(m, n)+\varepsilon} \\
& \text { i.e., } \log { }^{[p+1]} \mu\left(\exp ^{[n-1]} r, f \circ g\right) \leqslant \exp ^{[m-q-2]} r^{\rho_{g}(m, n)+\varepsilon} \\
& \text { i.e., } \quad \log [p+m-q-1] \mu\left(\exp ^{[n-1]} r, f \circ g\right) \leqslant \log ^{[m-q-2]} \exp ^{[m-q-2]} r^{\rho_{g}(m, n)+\varepsilon} \\
& \text { i.e., } \quad \log [p+m-q-1] \mu\left(\exp ^{[n-1]} r, f \circ g\right) \leqslant r^{\rho_{g}(m, n)+\varepsilon} \text {. }
\end{aligned}
$$

Again for all sufficiently large values of $r$, we obtain that

$$
\begin{aligned}
\log ^{[p]} \mu\left(\exp ^{[q-1]} r, f\right) & \geqslant\left(\lambda_{f}(p, q)-\varepsilon\right) \log [q] \exp ^{[q-1]} r \\
\text { i.e., } \quad \log ^{[p]} \mu\left(\exp ^{[q-1]} r, f\right) & \geqslant\left(\lambda_{f}(p, q)-\varepsilon\right) \log r \\
\text { i.e., } \quad \log [p] \mu\left(\exp ^{[q-1]} r, f\right) & \geqslant \log r^{\left(\lambda_{f}(p, q)-\varepsilon\right)} \\
\text { i.e., } \quad \log \left[{ }^{[p-1]} \mu\left(\exp ^{[q-1]} r, f\right)\right. & \geqslant r^{\left(\lambda_{f}(p, q)-\varepsilon\right)} .
\end{aligned}
$$

Now combining (11) of Case I and (15) we get for all sufficiently large values of $r$ that

$$
\frac{\log ^{[p]} \mu\left(\exp ^{[n-1]} r, f \circ g\right)}{\log ^{[p-1]} \mu\left(\exp ^{[q-1]} r, f\right)} \leq \frac{\left(\rho_{f}(p, q)+\varepsilon\right) r^{\left(\rho_{g}(m, n)+\varepsilon\right)}}{r^{\left(\lambda_{f}(p, q)-\varepsilon\right)}} .
$$

Now in view of (7) it follows from (16) that

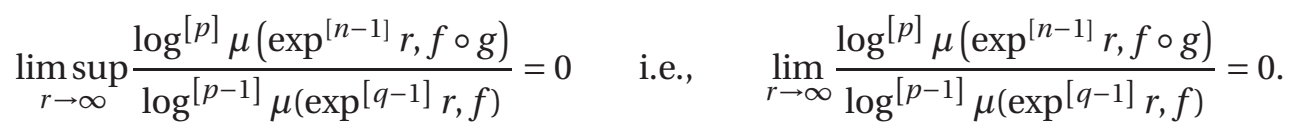

This proves the first part of the theorem.

Again combining (14) of Case II and (15) we obtain for all sufficiently large values of $r$ that

$$
\frac{\log ^{[p+m-q-1]} \mu\left(\exp ^{[n-1]} r, f \circ g\right)}{\log ^{[p-1]} \mu\left(\exp ^{[q-1]} r, f\right)} \leq \frac{r^{\rho_{g}(m, n)+\varepsilon}+O(1)}{r^{\left(\lambda_{f}(p, q)-\varepsilon\right)}} .
$$


Now in view of (7) it follows from (17) that $\limsup _{r \rightarrow \infty} \frac{\log ^{[p+m-q-1]} \mu\left(\exp ^{[n-1]} r, f \circ g\right)}{\log ^{[p-1]} \mu\left(\exp ^{[q-1]} r, f\right)}=0 \quad$ i.e., $\quad \lim _{r \rightarrow \infty} \frac{\log ^{[p+m-q-1]} \mu\left(\exp ^{[n-1]} r, f \circ g\right)}{\log ^{[p-1]} \mu\left(\exp ^{[q-1]} r, f\right)}=0$.

This establishes the second part of the theorem.

Remark 1. The condition $\rho_{g}(m, n)<\lambda_{f}(p, q)$ in Theorem 1 is essential as we see in the following example:

Example 1. Let $f=g=\exp z$ and $p=m=2, q=n=1$. Then

$$
\rho_{g}(m, n)=\lambda_{f}(p, q)=\rho_{f}(p, q)=1 .
$$

Now

$$
\begin{aligned}
\log \mu(r, f \circ g) & \geq \log M\left(\frac{r}{2}, f \circ g\right)+O(1) \geq T\left(\frac{r}{2}, f \circ g\right)+O(1) \\
& =T\left(\frac{r}{2}, \exp ^{[2]} z\right)+O(1) \sim \frac{\exp \left(\frac{r}{2}\right)}{\left(2 \pi^{3} \frac{r}{2}\right)^{\frac{1}{2}}}+O(1) \quad(r \rightarrow \infty)
\end{aligned}
$$

i.e., $\quad \log ^{[2]} \mu(r, f \circ g) \geq \frac{r}{2}-\frac{1}{2} \log r+O(1)$

$$
\text { and } \quad \log \mu(r, f) \leq \log M(r, f)=\log M(r, \exp z)=r \text {. }
$$

Then

$$
\begin{aligned}
\lim _{r \rightarrow \infty} \frac{\log ^{[p+m-q-1]} \mu\left(\exp ^{[n-1]} r, f \circ g\right)}{\log ^{[p-1]} \mu\left(\exp ^{[q-1]} r, f\right)} & =\lim _{r \rightarrow \infty} \frac{\log ^{[2]} \mu(r, f \circ g)}{\log \mu(r, f)} \\
& \geq \lim _{r \rightarrow \infty} \frac{\frac{r}{2}-\frac{1}{2} \log r+O(1)}{r} \\
& =\frac{1}{2} \neq 0 \text {, which is contrary to Theorem } 1 .
\end{aligned}
$$

Theorem 2. Let $f$ and $g$ be any two entire functions such that $\lambda_{g}(m, n)<\lambda_{f}(p, q) \leq \rho_{f}(p, q)<$ $\infty$, where $p, q, m, n$ are positive integers with $p>q, m>n$. Then

$$
\text { (i) } \quad \liminf _{r \rightarrow \infty} \frac{\log ^{[p]} \mu\left(\exp ^{[n-1]} r, f \circ g\right)}{\log ^{[p-1]} \mu\left(\exp ^{[q-1]} r, f\right)}=0 \quad \text { if } q \geq m
$$

and

$$
\text { (ii) } \quad \liminf _{r \rightarrow \infty} \frac{\log ^{[p+m-q-1]} \mu\left(\exp ^{[n-1]} r, f \circ g\right)}{\log ^{[p-1]} \mu\left(\exp ^{[q-1]} r, f\right)}=0 \quad \text { if } q<m \text {. }
$$

Proof. For a sequence of values of $r$ tending to infinity that

$$
\log ^{[m]} M\left(\exp ^{[n-1]} r, g\right) \leqslant\left(\lambda_{g}(m, n)+\varepsilon\right) \log ^{[n]} \exp ^{[n-1]} r
$$




$$
\begin{aligned}
\text { i.e., } \quad \log ^{[m]} M\left(\exp ^{[n-1]} r, g\right) & \leqslant\left(\lambda_{g}(m, n)+\varepsilon\right) \log r \\
\text { i.e., } \quad \log ^{[m]} M\left(\exp ^{[n-1]} r, g\right) & \leqslant \log r^{\lambda_{g}(m, n)+\varepsilon} \\
\text { i.e., } \quad \log ^{[m-1]} M\left(\exp ^{[n-1]} r, g\right) & \leqslant \log r^{\lambda_{g}(m, n)+\varepsilon} .
\end{aligned}
$$

Now from (9) and (18), we have for a sequence of values of $r$ tending to infinity that

$$
\log ^{[p]} \mu\left(\exp ^{[n-1]} r, f \circ g\right) \leqslant\left(\rho_{f}(p, q)+\varepsilon\right) r^{\lambda_{g}(m, n)+\varepsilon} .
$$

Combining (15) and (19) we get for a sequence of values of $r$ tending to infinity that

$$
\frac{\log ^{[p]} \mu\left(\exp ^{[n-1]} r, f \circ g\right)}{\log ^{[p-1]} \mu\left(\exp ^{[q-1]} r, f\right)} \leq \frac{\left(\rho_{f}(p, q)+\varepsilon\right) r^{\lambda_{g}(m, n)+\varepsilon}}{r^{\left(\lambda_{f}(p, q)-\varepsilon\right)}} .
$$

Now in view of (7) it follows from (20) that

$$
\liminf _{r \rightarrow \infty} \frac{\log [p] \mu\left(\exp ^{[n-1]} r, f \circ g\right)}{\log ^{[p-1]} \mu\left(\exp ^{[q-1]} r, f\right)}=0 .
$$

This proves the first part of the theorem.

Again for a sequence of values of $r$ tending to infinity that

$$
\begin{aligned}
\log ^{[m]} M\left(\exp ^{[n-1]} r, g\right) & \leqslant\left(\lambda_{g}(m, n)+\varepsilon\right) \log { }^{[n]} \exp ^{[n-1]} r \\
\text { i.e., } \quad \log ^{[m]} M\left(\exp ^{[n-1]} r, g\right) & \leqslant\left(\lambda_{g}(m, n)+\varepsilon\right) \log r \\
\text { i.e., } \quad \log ^{[m]} M\left(\exp ^{[n-1]} r, g\right) & \leqslant \log r^{\left(\lambda_{g}(m, n)+\varepsilon\right)} \\
\text { i.e., } \quad \exp ^{[m-q]} \log ^{[m]} M\left(\exp ^{[n-1]} r, g\right) & \leqslant \exp ^{[m-q]} \log r^{\left(\lambda_{g}(m, n)+\varepsilon\right)} \\
\text { i.e., } \quad \exp ^{[m-q]} \log ^{[m]} M\left(\exp ^{[n-1]} r, g\right) & \leqslant \exp ^{[m-q-1]} r^{\left(\lambda_{g}(m, n)+\varepsilon\right)} .
\end{aligned}
$$

Now from (12) and (21), we have for a sequence of values of $r$ tending to infinity that

$$
\begin{aligned}
& \log [p] \mu\left(\exp ^{[n-1]} r, f \circ g\right) \leqslant\left(\rho_{f}(p, q)+\varepsilon\right) \exp ^{[m-q-1]} r^{\left(\lambda_{g}(m, n)+\varepsilon\right)} \\
& \text { i.e., } \quad \log { }^{[p+1]} \mu\left(\exp ^{[n-1]} r, f \circ g\right) \leqslant \exp ^{[m-q-2]} r^{\left(\lambda_{g}(m, n)+\varepsilon\right)} \\
& \text { i.e., } \quad \log [p+m-q-1] \mu\left(\exp ^{[n-1]} r, f \circ g\right) \leqslant \log ^{[m-q-2]} \exp ^{[m-q-2]} r^{\left(\lambda_{g}(m, n)+\varepsilon\right)} \\
& \text { i.e., } \quad \log [p+m-q-1] \mu\left(\exp ^{[n-1]} r, f \circ g\right) \leqslant r^{\left(\lambda_{g}(m, n)+\varepsilon\right)} \text {. }
\end{aligned}
$$

Combining (15) and (22) we obtain for a sequence of values of $r$ tending to infinity that

$$
\frac{\log ^{[p+m-q-1]} \mu\left(\exp ^{[n-1]} r, f \circ g\right)}{\log ^{[p-1]} \mu\left(\exp ^{[q-1]} r, f\right)} \leq \frac{r^{\lambda_{g}(m, n)+\varepsilon}}{r^{\lambda_{f}(p, q)-\varepsilon}} .
$$

Now in view of (7) it follows from (23) that

$$
\liminf _{r \rightarrow \infty} \frac{\log ^{[p+m-q-1]} \mu\left(\exp ^{[n-1]} r, f \circ g\right)}{\log ^{[p-1]} \mu\left(\exp ^{[q-1]} r, f\right)}=0 .
$$

This establishes the second part of the theorem. 
Remark 2. The condition $\lambda_{g}(m, n)<\lambda_{f}(p, q)$ in Theorem 2 is necessary which is evident from the following example:

Example 2. Let $f=g=\exp z$ and $p=m=2, q=n=1$. Then

$$
\lambda_{g}(m, n)=\lambda_{f}(p, q)=\rho_{f}(p, q)=1
$$

Now

and

$$
\begin{aligned}
\log \mu(r, f \circ g) & \geq \log M\left(\frac{r}{2}, f \circ g\right)+O(1) \geq T\left(\frac{r}{2}, f \circ g\right)+O(1) \\
& =T\left(\frac{r}{2}, \exp ^{[2]} z\right)+O(1) \sim \frac{\exp \left(\frac{r}{2}\right)}{\left(2 \pi^{3} \frac{r}{2}\right)^{\frac{1}{2}}}+O(1)(r \rightarrow \infty)
\end{aligned}
$$

$$
\log \mu(r, f) \leq \log M(r, f)=\log M(r, \exp z)=r
$$

Therefore

$$
\begin{aligned}
\liminf _{r \rightarrow \infty} \frac{\log [p+m-q-1]}{\log ^{[p-1]} \mu\left(\exp ^{[n-1]} r, f \circ g\right)} & =\liminf _{r \rightarrow \infty} \frac{\log ^{[2]} \mu(r, f \circ g)}{\log \mu(r, f)} \\
& \geq \liminf _{r \rightarrow \infty} \frac{\frac{r}{2}-\frac{1}{2} \log r+O(1)}{r} \\
& =\frac{1}{2} \neq 0, \text { which is contrary to Theorem } 2 .
\end{aligned}
$$

Theorem 3. Let $f$ and $g$ be any two entire functions such that

(A) $\rho_{g}<\infty,(B) \lambda_{f}^{[l]}>0$ and $(C) \lambda_{f \circ g}(a, b)>0$ where $l, a, b$ are all positive integers with $l \geq 2$ and $a>b$. Also let $A<\rho_{g}$. Then for any two positive integers $m, n$ such that $m-n=1$ and $m>2$,

$$
\limsup _{r \rightarrow \infty} \frac{\log ^{[a]} \mu\left(\exp ^{[b-1]} r, f \circ g\right) \log ^{[l]} \mu(r, f \circ g)}{\left\{\log ^{[m]} \mu\left(\exp ^{[n]}\left(r^{A}\right), g\right)\right\}\left\{\log ^{[m]} \mu\left(\exp ^{[n-1]} r, g\right)\right\}}=\infty
$$

Proof. Let us choose $0<\varepsilon<\min \left\{\lambda_{f}^{[l]}, \lambda_{f \circ g}(a, b), \rho_{g}\right\}$.

Now from Lemma 1, we get for a sequence of values of $r$ tending to infinity that

$$
\begin{aligned}
\log ^{[l]} \mu(r, f \circ g) & \geq \log ^{[l]} \mu\left(\frac{1}{8} \mu\left(\frac{r}{4}, g\right)-|g(0)|, f\right)+O(1) \\
\text { i.e., } \quad \log ^{[l]} \mu(r, f \circ g) & \geq\left(\lambda_{f}^{[l]}-\varepsilon\right) \log \mu\left(\frac{r}{4}, g\right)+O(1) \\
\text { i.e., } \quad \log ^{[l]} \mu(r, f \circ g) & \geq\left(\lambda_{f}^{[l]}-\varepsilon\right)\left(\frac{r}{4}\right)^{\rho_{g}-\varepsilon} .
\end{aligned}
$$

Since $\rho_{g}(m, n)=1$, in view of Lemma 3 it follows for all sufficiently large values of $r$ that

$$
\log ^{[m]} \mu\left(\exp ^{[n]}\left(r^{A}\right), g\right) \leq(1+\varepsilon) \log ^{[n]} \exp ^{[n]}\left(r^{A}\right)
$$




$$
\text { i.e., } \quad \log ^{[m]} \mu\left(\exp ^{[n]}\left(r^{A}\right), g\right) \leq(1+\varepsilon)\left(r^{A}\right) \text {. }
$$

Now from (24) and (25), we get for a sequence of values of $r$ tending to infinity that

$$
\frac{\log ^{[l]} \mu(r, f \circ g)}{\log ^{[m]} \mu\left(\exp ^{[n]}\left(r^{A}\right), g\right)} \geq \frac{\left(\lambda_{f}^{[l]}-\varepsilon\right)\left(\frac{r}{4}\right)^{\rho_{g}-\varepsilon}}{(1+\varepsilon)\left(r^{A}\right)} .
$$

Again for all sufficiently large values of $r$,

$$
\log ^{[a]} \mu\left(\exp ^{[b-1]} r, f \circ g\right) \geq\left(\lambda_{f \circ g}(a, b)-\varepsilon\right) \log r
$$

and

$$
\log ^{[m]} \mu\left(\exp ^{[n-1]} r, g\right) \leq(1+\varepsilon) \log r .
$$

Therefore from the above two inequalities we get for all sufficiently large values of $r$ that

$$
\frac{\log ^{[a]} \mu\left(\exp ^{[b-1]} r, f \circ g\right)}{\log ^{[m]} \mu\left(\exp ^{[n-1]} r, g\right)} \geq \frac{\lambda_{f \circ g}(a, b)-\varepsilon}{1+\varepsilon} .
$$

Since $\varepsilon(>0)$ is arbitrary, it follows from above that

$$
\liminf _{r \rightarrow \infty} \frac{\log ^{[a]} \mu\left(\exp ^{[b-1]} r, f \circ g\right)}{\log ^{[m]} \mu\left(\exp ^{[n-1]} r, g\right)} \geq \lambda_{f \circ g}(a, b) .
$$

As $A<\rho_{g}$, we can choose $\varepsilon$ in such a way that

$$
A<\rho_{g}-\varepsilon
$$

Now from (26) and (28) it follows that

$$
\limsup _{r \rightarrow \infty} \frac{\log ^{[l]} \mu(r, f \circ g)}{\log ^{[m]} \mu\left(\exp ^{[n]}\left(r^{A}\right), g\right)}=\infty .
$$

Thus the theorem follows from (27) and (29).

Corollary 1. Under the same conditions of Theorem 3 when $m=2$

$$
\limsup _{r \rightarrow \infty} \frac{\log ^{[a]} \mu\left(\exp ^{[b-1]} r, f \circ g\right) \log ^{[l]} \mu(r, f \circ g)}{\left\{\log ^{[2]} \mu\left(\exp \left(r^{A}\right), g\right)\right\}\left\{\log ^{[2]} \mu(r, g)\right\}}=\infty \text {. }
$$

Remark 3. The condition $\lambda_{f}^{[l]}>0$ in Corollary 1 is essential as we see in the following example:

Example 3. Let

$$
f=z \text { and } g=\exp z
$$

Also let

$$
l=a=2 \text { and } b=1 \text {. }
$$


Then

$$
\lambda_{f}=0 \text { and } \rho_{g}=\lambda_{f \circ g}=1
$$

Now

$$
\log ^{[2]} \mu(r, f \circ g) \leq \log ^{[2]} M(r, f \circ g)=\log ^{[2]} M(r, g)=\log r
$$

and

$$
\log ^{[2]} \mu(r, g) \geq \log ^{[2]} M\left(\frac{r}{2}, g\right)=\log r+O(1)
$$

Hence

$$
\begin{aligned}
\limsup _{r \rightarrow \infty} \frac{\left\{\log ^{[2]} \mu(r, f \circ g)\right\}^{2}}{\log ^{[2]} \mu\left(\exp \left(r^{A}\right), g\right) \log ^{[2]} \mu(r, g)} & \leq \limsup _{r \rightarrow \infty} \frac{\{\log r\}^{2}}{\frac{r^{A}}{2}\{\log r+O(1)\}} \\
& =0, \quad \text { which is contrary to Corollary } 1 .
\end{aligned}
$$

Theorem 4. Let $f$ be an entire function such that $0<\lambda_{f}(p, q) \leq \rho_{f}(p, q)<\infty$. Also let $g$ be an entire function with $\rho_{g}^{[l]}>0$. Then

(i) $\quad \limsup _{r \rightarrow \infty} \frac{\log ^{[p]} \mu(r, f \circ g)}{\log ^{[p]} \mu\left(\exp ^{[l-1]}\left(r^{\mu}\right), f\right)}=\infty \quad$ if $q=l-1$

(ii) $\quad \limsup _{r \rightarrow \infty} \frac{\log ^{[p]} \mu(r, f \circ g)}{\log [p] \mu\left(\exp ^{[l-1]}\left(r^{\mu}\right), f\right)} \geq \frac{\rho_{g}^{[l]} \lambda_{f}(p, q)}{\mu \rho_{f}(p, q)} \quad$ if $q=l$

and

$$
\text { (iii) } \quad \limsup _{r \rightarrow \infty} \frac{\log ^{[p]} \mu(r, f \circ g)}{\log ^{[p]} \mu\left(\exp ^{[l-1]}\left(r^{\mu}\right), f\right)} \geq \frac{\lambda_{f}(p, q)}{\rho_{f}(p, q)} \quad \text { if } q>l
$$

where $\mu<\rho_{g}^{[l]}$ and $p, q, l$ are positive integers with $p>q$.

Proof. By Lemma 1 we obtain for a sequence of values of $r$ tending to infinity,

$$
\begin{array}{cl}
\log ^{[p]} \mu(r, f \circ g) \geq \log ^{[p]} \mu\left(\frac{1}{8} \mu\left(\frac{r}{4}, g\right), f\right)+O(1) \\
\text { i.e., } \quad \log [p] \mu(r, f \circ g) \geq\left(\lambda_{f}(p, q)-\varepsilon\right) \log ^{[q]} \mu\left(\frac{r}{4}, g\right)+O(1) \\
\text { i.e., } \quad \log ^{[p]} \mu(r, f \circ g) \geq\left(\lambda_{f}(p, q)-\varepsilon\right) \log ^{[q-l+1]} \log ^{[l-1]} \mu\left(\frac{r}{4}, g\right)+O(1) \\
\text { i.e., } \quad \log ^{[p]} \mu(r, f \circ g) \geq\left(\lambda_{f}(p, q)-\varepsilon\right) \log ^{[q-l+1]}\left(\frac{r}{4}\right)^{\left(\rho_{g}^{[l]}-\varepsilon\right)}+O(1) .
\end{array}
$$

Again from the definition of $\rho_{f}(p, q)$ it follows for all sufficiently large values of $r$ that

$$
\begin{gathered}
\quad \log { }^{[p]} \mu\left(\exp ^{[l-1]}\left(r^{\mu}\right), f\right) \leq\left(\rho_{f}(p, q)+\varepsilon\right) \log ^{[q]} \exp ^{[l-1]}\left(r^{\mu}\right) \\
\text { i.e., } \quad \log ^{[p]} \mu\left(\exp ^{[l-1]}\left(r^{\mu}\right), f\right) \leq\left(\rho_{f}(p, q)+\varepsilon\right) \log ^{[q-l+1]}\left(r^{\mu}\right) .
\end{gathered}
$$


Thus from (30) and (31) we have for a sequence of values of $r$ tending to infinity that

$$
\frac{\log ^{[p]} \mu(r, f \circ g)}{\log ^{[p]} \mu\left(\exp ^{[l-1]}\left(r^{\mu}\right), f\right)} \geq \frac{\left(\lambda_{f}(p, q)-\varepsilon\right) \log ^{[q-l+1]}\left(\frac{r}{4}\right)^{\left(\rho_{g}^{[l]}-\varepsilon\right)}+O(1)}{\left(\rho_{f}(p, q)+\varepsilon\right) \log ^{[q-l+1]}\left(r^{\mu}\right)} .
$$

Since $\mu<\rho_{g}^{[l]}$, the theorem follows from (32).

Remark 4. The condition $\mu<\rho_{g}$ in Theorem 4 is essential as we see in the following example:

Example 4. Let $f=g=\exp z$ and $p=m=2, q=n=1, l=2$. Also let $\mu=1$. Then

$$
\lambda_{f}(p, q)=\rho_{f}(p, q)=1 \text { and } \rho_{g}^{[l]}=\rho_{g}=1 .
$$

Now

$$
\log \mu(r, f \circ g) \leq \log M(r, f \circ g) \leq 3 T(2 r, f \circ g) \sim \frac{3 \exp (2 r)}{\left(4 \pi^{3} r\right)^{\frac{1}{2}}} \quad(r \rightarrow \infty)
$$

and

$$
\mu(\exp r, f) \leq M(\exp r, f)=\exp ^{[2]} r .
$$

Therefore

$$
\begin{aligned}
\limsup _{r \rightarrow \infty} \frac{\log [p] \mu(r, f \circ g)}{\log [p] \mu\left(\exp ^{[l-1]}\left(r^{\mu}\right), f\right)} & =\limsup _{r \rightarrow \infty} \frac{\log ^{[2]} \mu(r, f \circ g)}{\log ^{[2]} \mu(\exp r, f)} \\
& \leq \frac{2 r-\frac{1}{2} \log r+O(1)}{r} \\
& =2 \neq \infty, \text { which is contrary to Theorem } 4 .
\end{aligned}
$$

Theorem 5. Let $f$ and $g$ be any two entire functions such that $0<\lambda_{f}(p, q) \leq \rho_{f}(p, q)<\infty$ and $\rho_{g}(m, n)<\infty$, where $p, q, m, n$ are positive integers with $p>q, m>n$. Then

and

$$
\text { (i) } \quad \limsup _{r \rightarrow \infty} \frac{\log ^{[p+1]} \mu\left(\exp ^{[n-1]} r, f \circ g\right)}{\log ^{[p]} \mu\left(\exp ^{[q-1]} r, f\right)} \leqslant \frac{\rho_{g}(m, n)}{\lambda_{f}(p, q)} \quad \text { if } q \geq m
$$

$$
\text { (ii) } \quad \limsup _{r \rightarrow \infty} \frac{\log ^{[p+m-q]} \mu\left(\exp ^{[n-1]} r, f \circ g\right)}{\log ^{[p]} \mu\left(\exp ^{[q-1]} r, f\right)} \leqslant \frac{\rho_{g}(m, n)}{\lambda_{f}(p, q)} \quad \text { if } q<m \text {. }
$$

Proof. We have for all sufficiently large values of $r$

$$
\begin{aligned}
& \log { }^{[p]} \mu\left(\exp ^{[q-1]} r, f\right) \geq\left(\lambda_{f}(p, q)-\varepsilon\right) \log [q] \exp ^{[q-1]} r \\
\text { i.e., } & \log ^{[p]} \mu\left(\exp ^{[q-1]} r, f\right) \geq\left(\lambda_{f}(p, q)-\varepsilon\right) \log r .
\end{aligned}
$$

Case I. If $q \geqslant m$, then from (11) and (33) we get for all sufficiently large values of $r$ that

$$
\frac{\log ^{[p+1]} \mu\left(\exp ^{[n-1]} r, f \circ g\right)}{\log ^{[p]} \mu\left(\exp ^{[q-1]} r, f\right)} \leqslant \frac{\left(\rho_{g}(m, n)+\varepsilon\right) \log r+O(1)}{\left(\lambda_{f}(p, q)-\varepsilon\right) \log r}
$$




$$
\text { i.e., } \quad \limsup _{r \rightarrow \infty} \frac{\log ^{[p+1]} \mu\left(\exp ^{[n-1]} r, f \circ g\right)}{\log ^{[p]} \mu\left(\exp ^{[q-1]} r, f\right)} \leqslant \frac{\rho_{g}(m, n)+\varepsilon}{\lambda_{f}(p, q)-\varepsilon} .
$$

Since $\varepsilon(>0)$ is arbitrary, it follows from above that

$$
\limsup _{r \rightarrow \infty} \frac{\log ^{[p+1]} \mu\left(\exp ^{[n-1]} r, f \circ g\right)}{\log ^{[p]} \mu\left(\exp ^{[q-1]} r, f\right)} \leqslant \frac{\rho_{g}(m, n)}{\lambda_{f}(p, q)} .
$$

This proves the first part of the theorem.

Case II. If $q<m$ then from (14) and (33) we obtain for all sufficiently large values of $r$ that

$$
\begin{aligned}
\frac{\log [p+m-q]}{\left[\exp ^{[n-1]} r, f \circ g\right)} & \leqslant \frac{\left(\rho_{g}(m, n)+\varepsilon\right) \log r+O(1)}{\left(\lambda_{f}(p, q)-\varepsilon\right) \log r} \\
\text { i.e., } \limsup _{r \rightarrow \infty}^{[p]} \mu\left(\exp ^{[q-1]} r, f\right) & \log ^{[p+m-q]} \mu\left(\exp ^{[n-1]} r, f \circ g\right) \\
\log ^{[p]} \mu\left(\exp ^{[q-1]} r, f\right) & \leqslant \frac{\rho_{g}(m, n)+\varepsilon}{\lambda_{f}(p, q)-\varepsilon} .
\end{aligned}
$$

As $\varepsilon(>0)$ is arbitrary, it follows from above that

$$
\limsup _{r \rightarrow \infty} \frac{\log [p+m-q]}{\log ^{[p]} \mu\left(\exp ^{[n-1]} r, f \circ g\right)} \leqslant \frac{\rho_{g}(m, n)}{\lambda_{f}(p, q)} .
$$

Thus the second part of the theorem is established.

Remark 5. The condition $\rho_{g}(m, n)<\infty$ in Theorem 5 is necessary which is evident from the following example:

Example 5. Let $f=\exp z, g=\exp ^{[2]} z$ and $p=m=2, q=n=1$. Then

$$
\lambda_{f}(p, q)=\rho_{f}(p, q)=1 \text { and } \rho_{g}(m, n)=\infty
$$

Now

and

$$
\begin{array}{ll} 
& \log ^{[3]} \mu(r, f \circ g) \geq \log ^{[3]} M\left(\frac{r}{2}, f \circ g\right)+O(1) \\
\text { i.e., } & \log ^{[3]} \mu(r, f \circ g) \geq \log ^{[3]} M\left(\frac{r}{2}, f \circ g\right)+O(1) \\
\text { i.e., } & \log ^{[3]} \mu(r, f \circ g) \geq \log ^{[3]} \exp ^{[3]}\left(\frac{r}{2}\right)+O(1) \\
\text { i.e., } & \log ^{[3]} \mu(r, f \circ g) \geq\left(\frac{r}{2}\right)+O(1)
\end{array}
$$

$$
\log ^{[2]} \mu(r, f) \leq \log ^{[2]} M(r, f)=\log r
$$

Therefore

$$
\limsup _{r \rightarrow \infty} \frac{\log [p+m-q]}{\log ^{[p]} \mu\left(\exp ^{[n-1]} r, f \circ g\right)}=\limsup _{r \rightarrow \infty} \frac{\left.\log ^{[q-1]} r, f\right)}{\log ^{[2]} \mu(r, f \circ g)}
$$




$$
\begin{aligned}
& \text { i.e., } \quad \limsup _{r \rightarrow \infty} \frac{\log ^{[p+m-q]} \mu\left(\exp ^{[n-1]} r, f \circ g\right)}{\log ^{[p]} \mu\left(\exp ^{[q-1]} r, f\right)} \geq \limsup _{r \rightarrow \infty} \frac{\frac{r}{2}+O(1)}{\log r} \\
& \text { i.e., } \quad \limsup _{r \rightarrow \infty} \frac{\log ^{[p+m-q]} \mu\left(\exp ^{[n-1]} r, f \circ g\right)}{\log ^{[p]} \mu\left(\exp ^{[q-1]} r, f\right)}=\infty,
\end{aligned}
$$

which is contrary to Theorem 5 .

\section{References}

[1] J. Clunie, The composition of entire and meromorphic functions, Mathematical Essays dedicated to A. J. Macintyre, Ohio University Press (1970), 75-92.

[2] O. P. Juneja, G. P. Kapoor and S. K. Bajpai, On the (p,q)-order and lower (p,q)-order of an entire function, J. Reine Angew. Math., Vol. 282(1976), 53-67.

[3] D. Sato, On the rate of growth of entire functions of fast growth, Bull. Amer. Math. Soc., 69 (1963), 411-414.

[4] A. P. Singh, On maximum term of composition of entire functions, Proc. Nat. Acad. Sci. India, 59(A), Part I (1989), 103-115.

[5] A. P. Singh and M. S. Baloria, On maximum modulus and maximum term of composition of entire functions, Indian J. Pure Appl. Math., 22 (1991), 1019-1026.

[6] G. Valiron, Lectures on the General Theory of Integral Functions, Chelsea Publishing Company, 1949.

Department of Mathematics,University of Kalyani, Kalyani, Dist-Nadia,PIN- 741235, West Bengal, India.

E-mail: sanjib_kr_datta@yahoo.co.in

Rajbari, Rabindrapalli, R. N. Tagore Road, P.O. Krishnagar,Dist-Nadia,PIN- 741101, West Bengal, India.

E-mail: Tanmaybiswas_math@rediffmail.com; Tanmaybiswas_math@yahoo.com

Barabilla High School, P.O. Haptiagach, Dist-Uttar Dinajpur, PIN- 733202, West Bengal, India.

E-mail: manab_biswas83@yahoo.com 